\title{
Natural orifice transluminal endoscopic surgery
}

\section{T ARULAMPALAM, S PATTERSON-BROWN, AJ MORRIS, MC PARKER}

On behalf of: Association of Laparoscopic Surgeons of Great Britain and Ireland (ALSGBI); Association of Coloproctology of Great Britain \& Ireland (ACPGBI); Association of Upper Gastrointestinal Surgeons of Great Britain and Ireland (AUGIS); British Association of Urological Surgeons (BAUS); British Society of Gynaecological Endoscopy (BSGE); British Obesity \& Metabolic Surgical Society (BOMSS); and British Society of Gastroenterology (BSG)

Natural orifice transluminal endoscopic surgery (NOTES) has generated healthy and vigorous debate about the introduction of an entirely novel method of surgical therapy. Although there are many reasons for scepticism, there is undoubted interest in this field from both the medical profession and general public. Those Associations currently involved in laparoscopic and endoscopic surgery wish to safeguard patients and the reputation of the profession by issuing clear guidance and support for those wishing to undertake NOTES. The purpose of this document is to review the current status of both NOTES and hybrid NOTES, while at the same time identifying obstacles in both clinical research and training. Furthermore, it aims to provide a consensus statement on behalf of the main UK specialty associations involved in this field of surgery. The primary aim of this consensus statement is to provide a framework within which to develop, safely and effectively, what must still be considered an experimental technique.

\section{KEYWORDS}

Consensus statement - NOTES - Safe practice

\section{CORRESPONDENCE TO}

Tan Arulampalam, Laparoscopic General and Colorectal Surgeon. Colchester General Hospital, Turner Road, Colchester, Essex CO4 5JL, UK

E: laptan1@yahoo.co.uk

Advances in therapeutic endoscopy and minimally invasive surgery over the last decade have led to a convergence of techniques available for the treatment of a number of conditions. It is now possible to treat a wide variety of diseases using complex laparoscopic techniques including gallstones, gastro-oesophageal reflux disease (GORD) and obesity, colorectal cancer, many urological conditions and complex gynaecological problems. In parallel, the development of multichannel, flexible endoscopes with novel image manipulation including narrow band imaging and autofluorescence have led to the reality of more advanced therapeutic endoscopy such as endoscopic mucosal resection and submucosal dissection. These techniques allow endoscopic treatment of more diseases, including early cancer, than previously possible.

The natural development of these techniques was the possibility of major intra-abdominal interventions being performed through naturally occurring orifices offering surgery without skin incisions using a combination of laparoscopic and endoscopic techniques. The Society of American Gastrointestinal Endoscopic Surgeons (SAGES) and American Society of Gastrointestinal Endoscopy (ASGE) came together to form a working group as early as 2005, which has become known as the Natural Orifice Surgery Consortium for Assessment and Research (NOSCAR). ${ }^{1}$ They proposed the term for surgery performed using instruments which gain access through a natural orifice as NOTES (natural orifice transluminal endoscopic surgery).

NOTES procedures are performed using instruments in one body cavity, usually the peritoneal cavity, having gained access to this space by means of a natural orifice rather than percutaneously. Hybrid NOTES procedures are those which combine a NOTES approach as well as direct transcutaneous access to the cavity, usually in combination with laparoscopic instrumentation.

Initial NOTES developments were restricted to animal models. In 2005, Kalloo et al. ${ }^{2}$ demonstrated long-term survival in a porcine model of fallopian tube ligation. Since then, there have been several reports of successful tubal ligation, cholecystectomy, gastrojejunostomy, splenectomy and oophorectomy. ${ }^{2-6}$ In addition, there have also been anecdotal reports of human transgastric appendicectomy (N Reddy, personal communication). In 2007, the first human transvaginal cholecystectomies were reported by Bessler et $a l_{.}{ }^{7}$ and Marescaux et $a .^{8}$ Subsequently, case series of a hybrid NOTES cholecystectomy and total NOTES cholecystectomy have been published. ${ }^{9,10}$ 
There is now considerable interest in NOTES in the UK. The purpose of this document is to review the current status of both NOTES and hybrid NOTES, while at the same time identifying obstacles in both clinical research and training. Furthermore it aims to provide a consensus statement on behalf of the main UK specialty associations involved in this field of surgery, namely the ALSGBI, ACPGBI, AUGIS, BAUS, BSGE, BOMSS and BSG (Appendix 1).

\section{Technique}

\section{Peritoneal access}

The development of a safe and reproducible technique for gaining access to the operative field is essential for human NOTES. The principles of safe access are: (i) minimal tissue injury; (ii) good exposure; (iii) safety (avoiding vascular and visceral injury); and (iv) ability to maintain a seal and manipulate the instrument.

The most favoured route at present appears to be the stomach and a modified Seldinger dilatation or percutaneous endoscopic gastrostomy (PEG) technique can be used. Another approach is through the vault of the vagina. Transrectal approaches can also be used with transanal endoscopic microsurgery (TEMS) instruments, but still require great care in both access and closure.

\section{Closure}

Closure of the vagina may be achieved reasonably safely using currently available endoscopic surgical techniques but the same cannot yet be said for closing the rectum or stomach wall. However, on-going research into closure devices using T-tags and a variety of mechanical devices is promising. ${ }^{11,12}$ Expertise in the full array of closure techniques available will be an essential prerequisite for the surgeon or gastroenterologist undertaking NOTES.

\section{Intraperitoneal complications}

Much of the current scepticism for NOTES lies in the fact that the technique causes iatrogenic injury with the risk of immediate or delayed complication. These risks include infection, bleeding, visceral injury and delayed anastomotic or entry site leaks with potentially disastrous results. Laboratory research suggests that the risk of introducing infection is small but, nevertheless, requires full quantification. Vascular injury during access or a procedure can be both unrecognised and difficult to control due to the position and orientation of the endoscope and the currently available haemostatic technology. Damage to viscera is also a recognised risk and both the ability to identify and then repair any injury also needs to be evaluated fully. ${ }^{13}$

Long-term complications such as adhesion formation in addition to post NOTES dyspareunia must also be evaluated.

\section{Technology}

\section{Multitasking platform}

Whilst existing flexible endoscope technology enables the operator to perform some NOTES procedures, it is as yet limited. The ultimate goal remains the design and production of a flexible endoscope or NOTES platform that is multichannelled, can bend in more than two axes but which can also be stabilised once the correct operating position has been reached in order to carry out the required procedure. Currently, operators manipulate the instruments with relatively poorly designed hand-piece ergonomics and these will need to be further refined for NOTES.

Once achieved, a solid platform should then be maintained in order to permit at least two instruments to be manipulated for safe surgical dissection. However, instruments and current accessories are not yet robust enough for the force required for dissection, retraction and triangulation. Synergies exist with some robotic instrumentation and robotic control will have some part to play in the design and use of instruments of the future.

Another challenge for the instrumentation relates to poor spatial orientation. This factor is dependent on the experience of the operator, on the region being dissected and visual cues that are present. Operator experience and training are crucial in safe delivery of NOTES. Surgeons who do not carry out a large number of endoscopies may be less familiar with the intraluminal endoscopic views, particularly during retroflexion. Likewise, the experienced gastroenterologist is likely to find the intraperitoneal views, triangulation or spatial orientation much more of a challenge than the laparoscopic surgeon.

\section{Suturing}

Two issues of suturing during NOTES deserve special mention. First, closure of the access site, previously discussed above, which must ensure no anastomotic breakdown and subsequent leak. Second, the need for suturing during the procedure, whether to secure haemostasis or complete the operation, must also be considered. Once more, the instrumentation currently available is not consistently capable of permitting safe suturing of tissues.

\section{Ethics and acceptability}

We recognise that there are already well tested and proven procedures available to treat many surgical conditions which might be suitable for NOTES. It is, therefore, essential that NOTES indications and techniques are developed in non-human models followed by human research projects with appropriate institutional and regional ethical committee approval before its role in clinical practice can be evaluated. Current technology and equipment are not yet suitable for 
day-to-day clinical practice in humans. Close collaboration with industry partners is key to the future development of NOTES, the emphasis being that this speciality should be clinically driven.

\section{Research}

Although there are now rapidly increasing publications on NOTES, it is imperative to emphasise the importance of high-quality research which is adequately funded. There is little doubt that working within a multidisciplinary research group will help to negotiate the considerable obstacles currently placed in the way of a 'NOTES' surgeon or gastroenterologist. It is, therefore, desirable that those wishing to develop a NOTES programme should have access to accredited animal laboratory facilities for the relevant research and all results made available through a research network. For the investigator starting NOTES, it is essential that further knowledge on outcome in animal research is available before this technique can be applied directly into human clinical studies. We support the establishment of a national register of those undertaking NOTES research.

\section{Training}

Training in NOTES will be a key issue in the future and the experience of the British Society of Gastroenterology in relation to endoscopic training and surgical bodies that provide laparoscopic courses are good models for future education. Once appropriate NOTES procedures and techniques are identified, it is likely that mandatory animal laboratory training would be a prerequisite of any training course and accreditation programme. This training could be undertaken by multidisciplinary groups to optimise outcomes. Criteria to allow transfer of NOTES techniques into patients will need to be established and closely supervised by the appropriate specialist organisations, following which mandatory data reporting requirements should be set up to monitor outcomes.

\section{Conclusions}

NOTES has caused the surgical and gastroenterological community to engage in healthy and vigorous debate about the introduction of an entirely novel method of surgical therapy. Although there are many reasons for scepticism, there is undoubted interest in this field from both the medical profession and general public. Those associations currently involved in laparoscopic and endoscopic surgery wish to safeguard patients and the reputation of the profession by issuing clear guidance and support for those wishing to undertake NOTES. There remain many unanswered questions with regards to the risks, true benefits and cost of
NOTES. These must be answered fully before NOTES can be introduced as an acceptable technique in patients. Appropriate clinical governance remains the highest priority; therefore, we recommend the following:

1. The establishment of a UK NOTES working group drawn from those with experience of advanced laparoscopic and/or endoscopic techniques (ALSGBI, ACPGBI, AUGIS, BAUS, BSGE, BOMSS and BSG) to facilitate registry of NOTES research and stimulate development and debate of NOTES techniques.

2. Investigators should work as part of a multidisciplinary team.

3. NOTES techniques and indications need to be thoroughly evaluated in animal models before clinical trials in humans.

4. Human NOTES procedures should only be evaluated and carried out using techniques and equipment shown to be safe and effective for the purpose proposed in the context of clinical trials.

5. Once NOTES techniques become accepted in clinical practice, they should be carried out by operators who can demonstrate training and competence in that technique.

6. Investigators should ideally become members of the proposed research and development framework or professional associations.

These recommendations should be seen as encouraging safe practice and not stifling innovation. The responsible development of this exciting new field could have many benefits to both advanced laparoscopic and therapeutic endoscopic techniques even without the early introduction of NOTES if closer collaboration is introduced between all those with an interest in this field. The primary aim of this consensus statement is to provide a framework within which to safely and effectively develop what must still be considered an experimental technique.

\section{References}

1. Rattner D, Kalloo AN and the SAGES/ASGE Working Group on Natural Orifice Translumenal Endoscopic Surgery. ASGE/SAGES Working Group on Natural Orifice Translumenal Endoscopic Surgery. Surg Endosc 2005; 20: 329-33.

2. Jagannath SB, Kantesovoy SV, Vaughn CA, Chung SSC, Cotton PB, Gostout CJ et al. Per oral transgastric endoscopic ligation of fallopian tubes with long term survival in porcine model. Gastrointest Endosc 2005; 61: 449-53.

3. Wagh MS, Merrifield BF, Thompson CC. Survival studies after transgastric 
oophorectomy and tubectomy in a porcine model Gastrointest Endosc 2006; 63: 473-8.

4. Kantesovoy SV, Hu B, Jagannath SB, Vaughn CA, Beitler DM Chung SSC et al. Transgastric endoscopic splenectomy: is it possible? Surg Endosc 2006; 20: 522-5.

5. Bergstrom M, Ikeda K, Swain P, Park PO. Transgastric anastomosis by using flexible endoscopy in a porcine model (with video). Gastrointest Endosc 2006; 63: 307-12.

6. Pai RD, Fong DG, Bundga ME, Odze RD, Rattner DW, Thompson CC. Transcolonic endoscopic cholecystectomy: a NOTES survival study in a porcine model (with video). Gastrointest Endosc 2006; 64: 428-34.

7. Bessler M, Stevens PD, Milone L, Parikh M, Fowler D. Transvaginal laparoscopically assisted endoscopic cholecystectomy: a hybrid approach to natural orifice surgery. Gastrointest Endosc 2007; 66: 1243-5.

8. Marescaux J, Dallemagne B, Perretta S, Wattiez A, Mutter D, Coumaros D. Surgery without scars: report of transluminal cholecystectomy in a human being. Arch Surg 2007; 142: 823-6.
9. Palanivelu C, Rajan PS, Rangarajan M, Parasarathi R, Senthilnathan $P$, Praveenraj P. Transumbilical flexible endoscopic cholecystectomy in humans: first feasibility study using a hybrid technique. Endoscopy 2008; 40: 428-32.

10. Cardoso Ramos A, Murakami A, Galvao Neto M, Santana Galvao M, Souza Silva AC, Gonzalo Canseco E et al. NOTES transvaginal video assisted cholecystectomy: first series. Endoscopy 2008; 40: 572-6.

11. Ryou M, Fong DG, Pai RD, Rattner DW, Thompson CC. Transluminal closure for NOTES: an ex vivo study comparing leak pressures of various gastrotomy and colostomy modalities. Endoscopy 2008; 40: 432-7.

12. Voermans RP, Worm AM, Van Berge Henegouwen MI, Breeveld P, Bemelman WA, Fockens P. In vitro comparison and evaluation of seven gastric closure modalities for natural orifice transluminal endoscopic surgery (NOTES). Endoscopy 2008; 40: 595-602.

13. Pham BV, Morgan K, Romagnuolo J, Glenn J, Bazaz S, Lawrence C, Hawes R. Pilot study of adhesion formation following colon perforation and repair in a pig model using transgastric, laparoscopic or open surgical repair. Endoscopy 2008; 40: 664-70.

\section{Appendix 1: Main UK specialty associations involved in NOTES}

ALSGBI Association of Laparoscopic Surgeons of Great Britain and Ireland

ACPGBI Association of Coloproctology of Great Britain \& Ireland

AUGIS Association of Upper Gastro-intestinal Surgeons of Great Britain and Ireland

BAUS British Association of Urological Surgeons

BSGE British Society of Gynaecological Endoscopy

BOMSS British Obesity \& Metabolic Surgical Society

BSG British Society of Gastroenterology 